\title{
Regulation of inducible BALT formation and contribution to immunity and pathology
}

\author{
SY Foo ${ }^{1}$ and S Phipps ${ }^{1,2}$
}

Inducible bronchus-associated lymphoid tissue (BALT) is an organized tertiary lymphoid structure that is not preprogrammed but develops in response to infection or under chronic inflammatory conditions. Emerging research has shown that iBALT provides a niche for T-cell priming and B-cell education to assist in the clearance of infectious agents, highlighting the prospect that iBALT may be engineered and harnessed to enhance protective immunity against respiratory pathogens. Although iBALT formation is associated with several canonical factors of secondary lymphoid organogenesis such as lymphotoxin- $\alpha$ and the homeostatic chemokines, CXCL13, CCL19, and CCL21, these cytokines are not mandatory for its formation, even though they influence its organization and function. Similarly, lymphoid tissueinducer cells are not a requisite of iBALT formation. In contrast, dendritic cells are emerging as pivotal players required to form and sustain the presence of iBALT. Regulatory T cells appear to be able to attenuate the development of iBALT, although the underlying mechanisms remain ill-defined. In this review, we discuss facets unique to iBALT induction, the cellular subsets, and molecular cues that govern this process, and the contribution of this ectopic structure toward the generation of immune responses in the pulmonary compartment.

\section{INTRODUCTION}

Bronchus-associated lymphoid tissue (BALT) was first identified and described as a constitutive structure in the lungs of rabbits more than 30 years ago. ${ }^{1}$ BALT was initially described as follicular aggregations primarily composed of lymphocytes located, in general, along the bifurcations of the upper bronchi and between an artery and a bronchus. ${ }^{2}$ It is now recognized that BALT is an organized structure with T- and B-cell areas, high endothelial venules (HEVs) in the T-cell zone, and an overlying lymphoepithelium containing cells similar to microfold cells of Peyer's patches (PPs). ${ }^{3,4}$ Follicular dendritic cells (FDCs) in the BALT present antigen to $\mathrm{B}$ cells, ${ }^{5}$ as well as providing costimulatory signals that enhance B-cell activation and proliferation in the germinal centers (GCs). ${ }^{6,7}$ The majority of cells within BALT

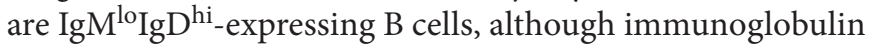
(Ig) $\mathrm{G}_{1^{-}}$, IgA,- and even IgE-positive plasma cells may also be observed depending on the nature of the microbe and/or antigen to which the cells are responding. ${ }^{8-11}$

BALT is considered to be part of the integrated mucosal system, which includes the gut-associated lymphoid tissue and nasal-associated lymphoid tissue (NALT) among others. It is regarded as a tertiary lymphoid tissue for unlike canonical secondary lymphoid organs (SLOs), BALT development is not pre-programmed, commences postnatally, and occurs anatomically at non-lymphoid locations. BALT formation can be induced under a range of disease states characterized by chronic inflammation, infection, or autoimmunity, ${ }^{12}$ and these structures are now preferentially termed inducible BALT (iBALT). They are distinct from the classical BALT in that they do not always possess an overlying epithelium, are not always found in association with an airway, and may form in the lung parenchyma.

The prevalence of BALT differs between species, being common in rabbits and rats and largely absent in healthy humans and mice. ${ }^{13,14}$ Emerging data now evidence a role of infectious agents suggesting that, like isolated lymphoid follicles (ILFs) in the gut, ${ }^{15}$ iBALT may develop in response to microbial exposure. However, it is noteworthy that BALT has been described in germ-free rats ${ }^{1}$ and mice. ${ }^{9}$

\section{DISEASE STATES}

Tertiary lymphoid organs (TLOs) are often noted in patients with chronic inflammatory diseases. These ectopic structures are characterized by distinct $\mathrm{T}$ - and $\mathrm{B}$-cell areas supported by specialized vascular systems that arise in atypical regions. ${ }^{16,17}$

\footnotetext{
${ }^{1}$ Centre for Asthma and Respiratory Diseases (CARD), School of Biomedical Sciences and Pharmacy, University of Newcastle, Newcastle, New South Wales, Australia.

${ }^{2}$ School of Biomedical Sciences, The University of Queensland, St Lucia, Queensland, Australia. Correspondence: S Phipps (s.phipps@uq.edu.au) 
TLOs have been shown to form in Sjogren's syndrome in both the lungs and salivary glands, ${ }^{18,19}$ in the lungs of subjects with idiopathic pulmonary fibrosis, ${ }^{20}$ and in the joints and lungs of individuals with rheumatoid arthritis (RA). ${ }^{19,21-23}$ Indeed, in the cohort of RA patients studied by Sato et al., ${ }^{23}$ BALT was observed in all eight subjects.

iBALT is frequently observed in lung tissue obtained from patients with asthma (another chronic inflammatory disease) and is evident in mouse models of experimental asthma. In a detailed clinical analysis, Elliot et al. ${ }^{24}$ revealed that cases with asthma tended to have larger and greater numbers of these aggregates as compared with non-asthma cases, regardless of asthma severity. The overexpression of interleukin (IL)- 5 in the mouse has been reported to promote iBALT, although whether this response is mediated through a direct effect on $\mathrm{B}$ cells remains to be determined. ${ }^{25}$ In a mouse model of allergic pulmonary inflammation, antigen-specific IgE-secreting plasma cells were shown to reside adjacent to the GCs in BALT. ${ }^{11}$ Clearly, reducing IgE synthesis is an attractive strategy for the treatment of asthma and other diseases in which IgE-mediated degranulation of mast cells and basophils contributes to disease pathogenesis.

Non-classical BALT lacking contact with the epithelium has also been observed in $84 \%$ of smokers and $14 \%$ of nonsmokers. ${ }^{26}$ Consistent with this, lymphoid follicles have been detected in the airways and parenchyma of mice exposed to cigarette smoke. ${ }^{27}$ These organized structures have also been noted in patients with chronic obstructive pulmonary disease (COPD), of which smoking is the leading cause. ${ }^{28}$ Respiratory viral infections, which have been implicated as a major risk factor for exacerbations of COPD, may also be causative agents of iBALT formation. Indeed, iBALT form within 10 days in mice following inoculation with influenza virus, even in the absence of the key cytokine lymphotoxin- $\alpha$ (LT- $\alpha){ }^{29}$ Administration of a replication-deficient strain of Vaccinia virus could also promote iBALT formation, ${ }^{30}$ suggesting that acute inflammation downstream of pattern recognition receptor (PRR) activation may be sufficient to initiate organogenesis. In this vein, Luhrmann et al. ${ }^{31}$ have demonstrated that rats repeatedly exposed to a toll-like receptor (TLR)2/6 ligand (MALP-2), developed larger BALT as compared with mice treated with control diluent alone. We and others have shown that a single exposure to the TLR4 ligand, lipopolysaccharide, is sufficient to drive the process of lymphoid neogenesis in the lungs of rats and mice $^{32}$ (S.Y. Foo, P.S. Foster and S. Phipps, unpublished data). A similar program appears to operate in the gut, where hundreds of ILFs are only induced after birth in response to the local commensal flora. ${ }^{33}$ Notably, the size and number of ILFs was observed to correlate with the size of the gut flora. ${ }^{15}$ Collectively, these findings suggest that iBALT (like other TLOs) develops under a number of pathological states and that the activation of PRR by infectious and non-infectious stimuli may be a common underlying cause of iBALT neogenesis.

\section{LTi CELLS AND iBALT FORMATION}

Many of the molecular and cellular requirements of SLO formation are also apparent during organogenesis of TLO. However, it is now emerging that a number of the cues that are indispensible for SLO formation are not absolutely necessary for iBALT formation. A greater understanding of distinct processes between SLO and iBALT formation will identify new targets for therapeutic intervention to either promote iBALT formation (e.g., for vaccine therapy) or resolution (e.g., to attenuate pathogenic autoimmune responses that may prosper in iBALT).

The classical model of lymphoid organogenesis for SLOs such as the lymph nodes (LNs) and PPs involves the interaction of a unique subset of hematopoietic cells, known as lymphoid tissue-inducer (LTi) cells, with local stromal organizer cells. This communication results in the upregulation of adhesion molecules such as vascular cellular adhesion molecule-1 (VCAM-1), intercellular adhesion molecule-1 (ICAM-1), and mucosal addressin cell adhesion molecule-1 (MAdCAM-1), as well as the secretion of a panel of lymphocyte-homing chemokines and pleiotropic cytokines.

$\mathrm{CD} 3{ }^{-} \mathrm{CD} 4{ }^{+} \mathrm{CD} 45^{+}$LTi cells are derived from fetal liver progenitors ${ }^{34}$ and are widely regarded as the earliest cell type to colonize the lymphoid anlagen of SLOs. It was recently determined that LTi cell differentiation is dependent upon at least two transcription factors, Id 2 and ROR- $\gamma$ t. Hence, LTi cells are absent in mice lacking Id 2 or ROR- $\gamma \mathrm{t}$, and this affects the development of peripheral LN, PPs, ILFs, and NALT, while splenic architecture is normal. ${ }^{35-37}$ LTi cells have been shown to accumulate in developing NALT and the adoptive transfer of these cells to Id2-deficient mice reconstituted the formation of NALT. $^{38}$ The formation of other SLOs absent from Id2-deficient mice was not achieved using this protocol, as the transfer of cells occurred after birth, and therefore after the developmental window during which LN and PP formation is initiated. Thus, Id2-regulated LTi cells are the common ancestor (or inducer) cells for the organogenesis of NALT and PPs.

In contrast to SLO, limited evidence is available on the requirement for LTi cells in TLO formation. However, the observation that ectopic structures can form in the thyroid glands of Id2deficient mice ${ }^{39}$ suggests that other cellular subsets may be able to compensate for the instructive signals normally delivered by LTi cells. ${ }^{40}$ These include mature $\mathrm{CD} 3{ }^{+} \mathrm{CD} 4{ }^{+}$T lymphocytes, B cells expressing LT $\alpha 1 \beta 2,{ }^{41}$ and T cells expressing LIGHT (another ligand of LT- $\beta$ receptor (LT- $\beta$ R ))..$^{42}$ To date, iBALT formation has not been shown to require the recruitment of LTi cells. As such, the cells that constitute the anlagen and seed the development of the iBALT appear distinct from those that generate SLOs and ILFs, and their identity remains unknown at this time (Figure 1). The identification of the inducer population(s) and/or essential molecular pathway in iBALT formation could allow the selective induction or resolution of this ectopic structure as required.

\section{TNF FAMILY MEMBERS AND HOMEOSTATIC CHEMOKINES}

Selected members of the tumor necrosis factor (TNF) family have been shown to be essential for the formation and organization of SLOs-namely, LT- $\alpha / \beta$, LIGHT, as well as TNF- $\alpha$ itself. ${ }^{40,44,45}$ The LT $\alpha 1 \beta 2$ heterotrimer is expressed on the surface of LTi cells and upon engagement of the cognate receptor 


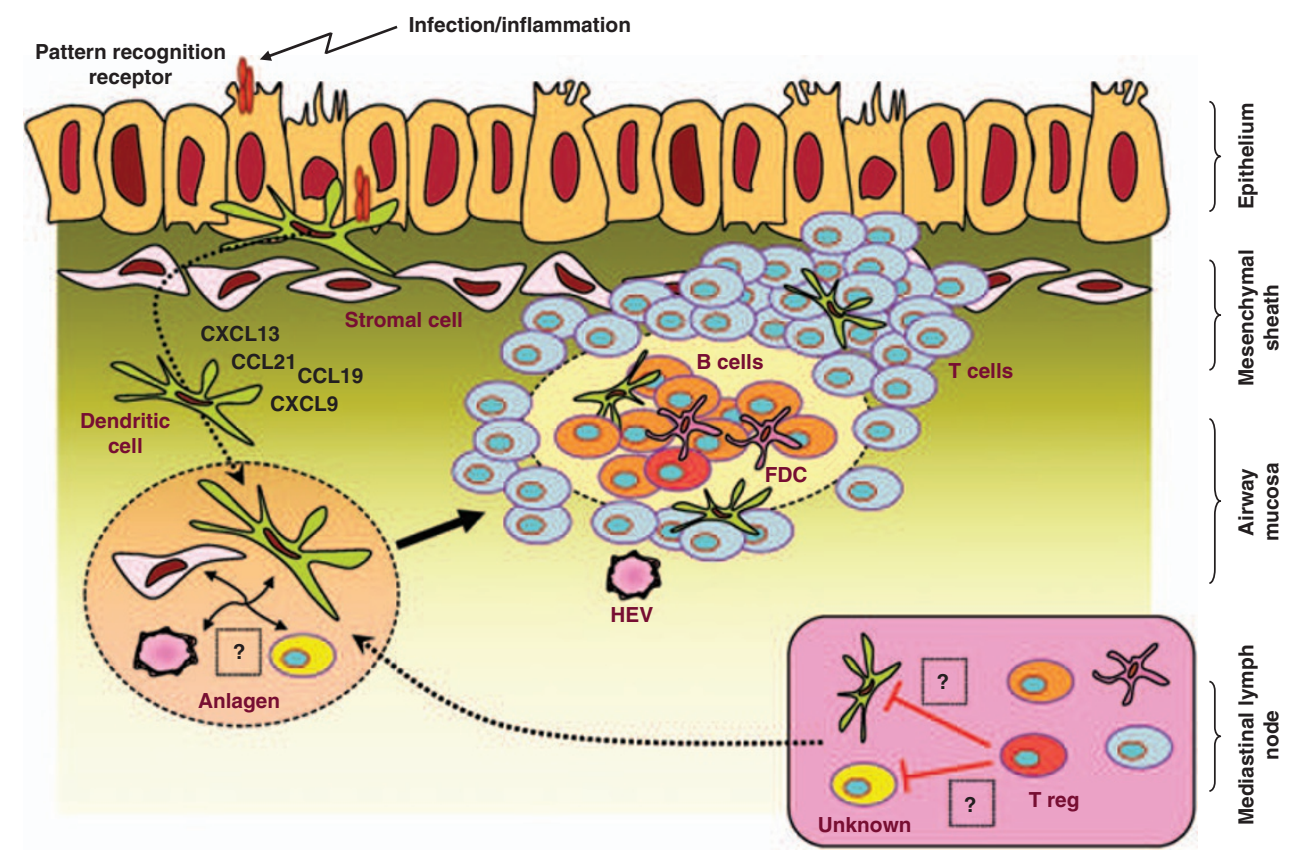

Figure 1 Under conditions of infection/inflammation, various antigens may stimulate PRRs (such as TLRs) on bronchoeptithelial cells or DCs. The early analgen that eventually gives rise to an iBALT depends on complex interactions between a number of cell types including activated DCs and stromal cells. Through the release of a host of chemokines, cytokines and the expresion of a unique combination of adhesion molecules on HEVs, $B$ and $T$ cells are recruited to the site and often organized into distinct zones. FDCs are found within the B-cell region where they present antigen and co-stimulatory signals to the B cells. It has been established that DCs are critical for the maintenance of iBALT structures. Tregs in the draining mediastinal LNs are able to attenuate iBALT formation, but the mechnism(s) by which they exert their suppressive effects from this remote location remains unknown. It is possible that Tregs my inhibit the activation/proliferation/exit of an as yet unidentified population of cells in the dLNs that then migrate to the lungs to initiate iBALT formation.

LT- $\beta$ R on stromal cells, triggers the production of the homeostatic chemokines CXCL13, CXCL12, CCL19, and CCL21. The resultant accumulation of $\mathrm{B}$ and $\mathrm{T}$ lymphocytes produces a second "wave" and rich supply of LT $\alpha 1 \beta 2$, which acts in a positive feedback loop to further enhance chemokine expression and cell recruitment. ${ }^{46,47}$ In support of LT activation of stromal cells, HEVs in LT-deficient mice lack the expression of key factors such as peripheral node addressin (PNAd), GlyCAM-1, and the enzyme HEC-6ST, which is required for the luminal expression of PNAd. ${ }^{48}$

Lymphocytes enter the BALT after first tethering to adhesion molecules, such as PNAd and VCAM-1, but not MAdCAM-1, expressed by HEVs. ${ }^{49,50}$ Notably, the strong luminal expression of VCAM-1 in BALT HEVs does not occur in SLOs. The L-selectinPNAd pathway and LFA-1 is crucial for lymphocyte homing, whereas $\alpha 4$ integrin and VCAM- 1 facilitate the recruitment of memory T cells to BALT. ${ }^{49}$ Taken together, these findings suggest that the HEVs in iBALT possess a unique pattern of adhesion molecule expression and therefore the entry requirements for lymphocytes to this compartment may be distinct from LNs and other SLOs.

One of the main challenges in the field of lymphoid neogenesis is to elucidate the carefully choreographed processes that direct cell recruitment and/or proliferation of many different cell types to generate a highly organized structure that is not pre-programmed. On the basis of a number of clinical studies documenting different lung pathologies, and animal work in factor-deficient and transgenic mice, it appears that at least three common events may initiate TLO formation: (i) activation of innate PRR, (ii) inflammatory cytokine (such as TNF- $\alpha$ or LT) and lymphoid chemokine production by hematopoietic or stromal cells, and (iii) activation/maturation of antigenpresenting cells and HEV development. For example, the size and complexity of TLOs in patients with autoimmune diseases has been shown to correlate with the expression of homeostatic chemokines and cytokines, in particular LT- $\alpha / \beta,{ }^{19}$ CXCL13, and CCL21. ${ }^{51,52}$ LT- $\alpha$ is critical to the development and organization of SLOs, as evidenced by the lack of all LNs, and PPs, and the presentation of abnormal splenic architecture in LT- $\alpha$-deficient mice. ${ }^{53,54}$ Loss of LT- $\beta$ R signaling via the administration of a blocking LT- $\beta$ R antibody in influenza-infected mice significantly decreased the number of iBALT structures and GC B cells. ${ }^{10}$ Despite this, the formation of iBALT has been observed in LT- $\alpha$-deficient mice in response to influenza infection, ${ }^{29}$ although these structures were poorly organized and lacked FDCs and HEVs expressing PNAd. These defects were overcome when LT- $\alpha$-deficient mice were reconstituted with wild-type bone marrow. Of note, LT- $\alpha$-deficient mice were still able to mount a delayed immune response and significantly, this was observed to be less pathological. This elegant study suggests that precise structural organization of iBALT is not absolutely necessary for its functionality and that iBALT may assist in the development and regulation of optimal protective immunity at the local tissue site.

The transgenic overexpression of TNF- $\alpha$ in the lung can result in the development of lymphoid organs both around the airways 
and in the parenchyma, ${ }^{55}$ consistent with the increased expression of TNF- $\alpha$ in patients with Sjogren's syndrome, COPD, and RA. The overexpression of IL- 6 together with its cognate receptor IL-6R also leads to iBALT formation. ${ }^{56}$ Although molecular insights into how either of these cytokines induce iBALT remain to be elucidated, both cytokines are potent inducers of homeostatic and inflammatory chemokines, which are likely to shape the early anlagen. CXCL13, for instance, is the archetypal $\mathrm{B}$-cell chemoattractant and serves to regulate the compartmentalization of CXCR5 ${ }^{+}$B cells within the follicle. ${ }^{57}$ CXCL13 has a critical role in the development of all LNs, although its ectopic expression leads to lymphoid neogenesis. ${ }^{58}$ CXCL13 also recruits LTi cells and T follicular helper (TFH) cells, ${ }^{59}$ and is primarily produced by FDCs. ${ }^{41}$ Unexpectedly, however, Rangel-Moreno et al..$^{43}$ have demonstrated that the absence of CXCL13 in the lung does not markedly affect iBALT formation in influenzainfected mice. These animals still developed well-organized follicles with segregated T- and B-cell areas, accompanied by HEVs and GCs, leading the authors to propose that other chemokines and/or as yet undiscovered ligands of CXCR5 may serve as a substitute for CXCL13.

Hyperplastic bronchial epithelial cells and T-cell zone fibroblastic reticular cells have been reported to express both the CCR7 ligands, CCL19 and CCL21. ${ }^{60,61}$ Stromal cells were also identified as being the major source of CCL21 in NALT. ${ }^{62}$ In the iBALT of both humans and mice, CCL21 was frequently localized to endothelial cells and reticular cells that were either on the periphery of follicles or in the interfollicular areas. ${ }^{19,29}$ Yet mice deficient in CCR7 spontaneously develop iBALT. ${ }^{9}$ Consistent with this, mice lacking both CCL19 and CCL21 (plt/plt mice) can develop iBALT with normal B-cell follicles. However, the $\mathrm{T}$-cell areas are poorly organized and as a consequence both $\mathrm{B}$ and T-cell responses are impaired. ${ }^{43}$ Collectively, these findings highlight the importance of iBALT organization and productive $\mathrm{T}$ cell help to ensure optimal BALT functionality.

It has been established that the respiratory epithelium may participate in inflammation in several ways, either as target cells that alter their function in response to a variety of biological mediators, or as effector cells that directly contribute to inflammation. For instance, pulmonary epithelial cells express an array of innate PRR including TLR1-963 and secrete a plethora of cytokines, chemokines, and growth factors upon activation. These factors may initiate adaptive immunity through the maturation and migration of immature dendritic cells (DCs). ${ }^{64}$ It seems likely that the epithelium has a significant role in iBALT formation given its location and ability to influence the local DC network, which is emerging as integral component to iBALT formation and persistence (see below), yet this has yet to be specifically examined.

\section{AGE DEPENDENCY}

It is notable that BALT has been observed in the lungs of up to $50 \%$ of healthy infants and young children, suggesting a predisposition in early life. ${ }^{65,66}$ Gould and Isaacson ${ }^{67}$ recorded the presence of BALT in human fetal and infant lungs and concluded that its appearance is probably dependent on antigenic stimulus, in agreement with the findings of others who had established an association between the amount of antigenic stimulation and BALT formation in humans. ${ }^{68,69}$

As the development of most SLOs is pre-programmed and occurs before birth, it has been hypothesized that TLOs would be more readily induced during a defined developmental window soon after birth, when the SLO are functionally immature. ${ }^{16}$ In support of this, the adoptive transfer of neonatal LN cells to the skin of neonatal mice was found to induce lymphoid structures displaying clear segregation of B- and T-cell areas, FDCs, and HEVs. ${ }^{70}$ By contrast, the administration of these cells to adult mice only generated disorganized aggregates of lymphocytes lacking FDCs. Remarkably, the organization of these ectopic structures was enhanced after the administration of an agonistic anti-CD40 antibody to mimic immune activation. This highlights the distinctive ability of neonates to develop more organized ectopic structures when compared with adult mice, in which the structural organization process is potentially antigen dependent. In a study designed to investigate the effect of age on allergic senitization, we observed that mice treated with lipopolysaccharide in the first week of life develop more iBALT than mice exposed at 4 weeks of age ${ }^{71}$ (S.Y. Foo, P.S. Foster and S. Phipps, unpublished data). Other studies reported differences in the leukocyte influx into the lung interstitium between rats of different ages after the intratracheal application of MALP-2. ${ }^{72,73}$ Taken together with clinical observations, these findings caution against generalizations of the immune responses between different age groups and suggest that susceptibility to develop iBALT is heightened in early life. However, the molecular pathways that underpin this phenomenon remain undefined.

\section{DENDRITIC CELLS CONTRIBUTE TO IBALT FORMATION AND PERSISTENCE}

CD $11 \mathrm{c}^{+}$conventional DCs (cDCs) and CD21 ${ }^{+}$FDCs are regular features of iBALT, with the former often interspersed within the T- and B-cell area and the latter usually found within B-cell follicles. ${ }^{19,29,74}$ Although a large body of literature exists on the antigen presentation abilities of DCs, ${ }^{75}$ less is known in regard to their function in orchestrating TLO development and organization. However, emerging evidence suggests that DCs are likely to be important organizers of iBALT formation as they are among the earliest cells to colonize the anlagen. In addition, two studies published in 2009 have demonstrated that DCs are necessary for the maintenance of iBALT in response to virus infection. ${ }^{10,30}$ Diptheria toxin-induced deletion of DCs after viral clearance led to a gradual decline in the number and size of iBALT structures. Strikingly, the lack of DCs coincided with the disappearance of virus-specific plasma cells and led to decreased IgA titers in the lung and virus-specific antibody titers in serum. Previous studies have implicated DCs as a potent source of inflammatory and homeostatic chemokines, ${ }^{76}$ and this was supported by GeurtsvanKessel et al. who demonstrated that the expression of key factors, such as CXCL12, CXCL13, CCL19, and CCL21, increased over time in lung DCs sorted from infected mice. Thus, in addition to presenting antigenic and costimulatory signals, DCs may promote the entrance and survival of 
lymphocytes within the iBALT. More recently, plasmacytoid DCs were found to accumulate in the lymphoid follicles of COPD patients. ${ }^{77}$ These were able to produce higher levels of TNF- $\alpha$ and IL-8 upon maturation as compared with plasmacytoid DCs from healthy subjects, but the role of these cells in iBALT formation and maintenance remains an open question.

\section{CONTRIBUTION OF REGULATORYT CELLSTO IBALT FORMATION}

In light of the findings that DCs contribute to the formation and maintenance of iBALT, it is probable that alterations in regulatory T-cell (Treg) migration and function may affect iBALT formation. Originally identified as $\mathrm{CD} 4{ }^{+} \mathrm{CD} 25^{+}$cells that were crucial for the prevention of autoimmune diseases, ${ }^{78}$ forkhead box P3 (Foxp3) (+) Tregs have since been demonstrated to mediate their tolerogenic effects by suppressing effector T-cell responses, either directly or by modulating DC immunogenicity. ${ }^{79-81}$ These effects are primarily mediated through the release of inhibitory cytokines such as IL-10 and TGF- $\beta$ or via contact-dependent mechanisms. ${ }^{82,83}$

The first study to directly implicate a role of Tregs arose from an observation that CCR7-deficient mice spontaneously developed iBALT from 5 days of age even in the absence of an environmental insult. ${ }^{9}$ Although not functionally impaired, Tregs were unable to home to the draining LN of CCR7-deficient mice. Tregs are known to express the homing receptor CCR7 at high levels ${ }^{84}$ and may exert their suppressive activity in the draining LN, reiterating the potential importance of site-specific regulation. ${ }^{42}$ Of note, the adoptive transfer of wild-type Tregs to CCR7-deficient recipients attenuated iBALT formation. Thus, Tregs control the development of iBALT formation and mediate at least part of their suppressive effects in the LN compartment. Further evidence to support the interrelationship between LNs and BALT formation was illustrated following the transplantation of human fetal bronchial tissue to SCID mice; only when the peripheral LNs were cotransplanted did the mice develop BALT. ${ }^{85}$ Further studies will be required to delineate the origin of the cells targeted by Tregs and the molecular processes that mediate this inhibition. Curiously, immunophenotyping of bronchial biopsy specimen from human infants has shown that Foxp ${ }^{+} \mathrm{CD} 4^{+}$Tregs reside primarily within these follicles. ${ }^{86}$ Intriguingly, Foxp $3^{+} \mathrm{CD} 4^{+} \mathrm{T}$ cells may also differentiate into $\mathrm{T}$ follicular helper cells and proceed to participate in the induction of GCs and local IgA synthesis. ${ }^{87}$ Although this phenomenon is reportedly restricted in the context of SLOs to PPs and not spleen or LNs, it is tempting to speculate that a similar scenario may occur in the context of iBALT.

\section{IBALT CONTRIBUTES TO IMMUNITY}

Elucidating the function(s) of TLOs, and in particular iBALT, remains an area of intense research. The initial concept of BALT being part of a common mucosal system ${ }^{88}$ was supported by the finding that gut-associated lymphoid tissue lymphocytes could populate the bronchial mucosa, and that the reverse was also true. ${ }^{89}$ Whether iBALT is beneficial or detrimental appears to be context dependent. Hogg et al. ${ }^{28}$ reported that the presence of lymphoid follicles resembling BALT correlates with disease severity in patients with COPD, whereas iBALT associates with local tissue pathology in the lungs of RA patients. ${ }^{19}$ In addition, BALT has been identified as a favorable site for pathogenic microorganisms during latency. ${ }^{90}$

In contrast, it has been proposed that iBALT may serve as an inducible lymphoid tissue for respiratory immune responses. To investigate this theory, Moyron-Quiroz et al..$^{29}$ examined the adaptive response of mice lacking some or all peripheral lymphoid organs following inoculation with influenza virus. Remarkably, mice without SLOs were still able to generate a robust virus-specific cytotoxic T-cell response and virus-specific antibody production because of the presence of iBALT. Moreover, these mice survived higher doses of the virus as compared with their wild-type counterparts. In a similar study, the iBALT observed in influenza-infected mice lacking spleens, LNs, and PPs was found up to 90 days after infection. ${ }^{74}$ The authors proposed that iBALT was able to support the proliferation of virus-specific $\mathrm{CD} 8+\mathrm{T}$ cells that then proceeded to clear a challenge infection. Further support of a protective role of iBALT has been provided in two recent studies that used microbial molecules to induce BALT before inoculation with a respiratory pathogen. Using mice deficient of peripheral lymphoid organs, Wiley et al. ${ }^{91}$ demonstrated that induction of iBALT formation with five doses of the non-infectious protein cage nanoparticle (PCN) over 12 days up till 3 days before infection accelerated the clearance of a variety of respiratory viruses. Importantly, PCN-treated mice displayed reduced lung damage after influenza virus infection as compared with control phosphate-buffered saline-treated mice. One significant caveat with these findings stems from separating the contribution of BALT per se vs. the adjuvant-inducing effects of the microbial ligands. It is noteworthy that increasing the length of time between PCN exposure and virus inoculation led to a dramatic fall off in efficacy. In another immunomodulatory study, iBALT was noted as early as 4 days after challenge in mice exposed to cholera toxin, a mutant form of the Escherichia coli heat-labile enterotoxin, or CpG oligodeoxynucleotides. ${ }^{92}$ Although the authors did not conduct further investigation into their role, they noted that the presence of iBALT correlated with improved survival when the mice were subsequently infected with a lethal dose of influenza virus.

More recently, Halle et al. ${ }^{30}$ have employed new technology to address the functionality of iBALT in an ex vivo culture system. Using two-photon microscopy, stable interactions between antigen-loaded DCs and antigen-specific T cells within iBALT were confirmed. These $\mathrm{T}$ cells were found to proliferate as well as those residing in the draining LN. As the antigen-specificity of the $T$ cells differed from the antigen used to elicit the formation of the iBALT in this model, their findings support the idea of iBALT functioning as a general priming site for $\mathrm{T}$ cell responses. Taken together, these studies lend support to the concept that iBALT induction would be an advantageous element of future immunoprophylactic strategies charged with enhancing protective immunity against respiratory pathogens.

\section{CONCLUDING REMARKS}

Converging evidence suggests that the formation of iBALT can be initiated through the activation of innate PRR. As ectopic 
structures, the induction of iBALT has been shown to modulate adaptive immunity, either by contributing to the generation of beneficial humoral and cellular immune responses or exacerbating local tissue damage in autoimmune diseases. As iBALT can serve as an effective antigen presentation site, the development of novel adjuvants to promote its formation may enhance current vaccination strategies. Alternatively, resolution of iBALT may be desirable in the treatment of COPD and autoimmune diseases. To this end, a better understanding of the events that orchestrate iBALT formation will help to identify novel targets for the design of therapies to promote iBALT formation or removal. Several questions remain unanswered, including the nature of the cells that initiate the anlagen, the contribution of epithelial-derived mediators and local DC networks, the role of $\mathrm{T}$ follicular helper cells in iBALT formation and function, and the mechanisms by which Tregs control iBALT organogenesis. Ultimately, further investigation will be required to fully elucidate the immune signals that control the formation of iBALT. As more details emerge, understanding how to harness the inherent plasticity of these lymphoid structures will open up new avenues in the fields of adjuvant biology and vaccine development.

\section{ACKNOWLEDGMENTS}

SYF is supported by a scholarship from the Priority Research Centre for Asthma and Respiratory Diseases, University of Newcastle. SP is supported by funding from the NHMRC of Australia.

\section{DISCLOSURE}

The authors declared no conflict of interest.

(c) 2010 Society for Mucosal Immunology

\section{REFERENCES}

1. Bienenstock, J., Johnston, N. \& Perey, D.Y. Bronchial lymphoid tissue. I. Morphologic characteristics. Lab. Invest. 28, 686-692 (1973).

2. Sminia, T., van der Brugge-Gamelkoorn, G.J. \& Jeurissen, S.H. Structure and function of bronchus-associated lymphoid tissue (BALT). Crit. Rev. Immunol. 9, 119-150 (1989).

3. Pankow, W. \& von Wichert, P. M cell in the immune system of the lung Respiration 54, 209-219 (1988).

4. Corr, S.C., Gahan, C.C. \& Hill, C. M-cells: origin, morphology and role in mucosal immunity and microbial pathogenesis. FEMS Immunol. Med. Microbiol. 52, 2-12 (2008).

5. Suzuki, K., Grigorova, I., Phan, T.G., Kelly, L.M. \& Cyster, J.G. Visualizing B cell capture of cognate antigen from follicular dendritic cells. J. Exp. Med. 206, 1485-1493 (2009).

6. van der Brugge-Gamelkoorn, G.J., van de Ende, M.B. \& Sminia, T. Nonlymphoid cells of bronchus-associated lymphoid tissue of the rat in situ and in suspension. With special reference to interdigitating and follicular dendritic cells. Cell Tissue Res. 239, 177-182 (1985).

7. Kosco-Vilbois, M.H., Gray, D., Scheidegger, D. \& Julius, M. Follicular dendritic cells help resting $B$ cells to become effective antigenpresenting cells: induction of B7/BB1 and upregulation of major histocompatibility complex class II molecules. J. Exp. Med. 178, 2055-2066 (1993).

8. Milne, R.W., Bienenstodk, J. \& Perey, D.Y. The influence of antigenic stimulation on the ontegeny of lymphoid aggregates and immunoglobulincontaining cells in mouse bronchial and intestinal mucosa. J. Reticuloendothel. Soc. 17, 361-369 (1975).

9. Kocks, J.R., Davalos-Misslitz, A.C.M., Hintzen, G., Ohl, L. \& Forster, R. Regulatory $T$ cells interfere with the development of bronchus-associated lymphoid tissue. J. Exp. Med. 204, 723-734 (2007).

10. GeurtsvanKessel, C.H. et al. Dendritic cells are crucial for maintenance of tertiary lymphoid structures in the lung of influenza virus-infected mice. J. Exp. Med. 206, 2339-2349 (2009).
11. Chvatchko, Y., Kosco-Vilbois, M.H., Herren, S., Lefort, J. \& Bonnefoy, J.Y. Germinal center formation and local immunoglobulin $E$ (IgE) production in the lung after an airway antigenic challenge. J. Exp. Med. 184, 2353-2360 (1996).

12. Aloisi, F. \& Pujol-Borrell, R. Lymphoid neogenesis in chronic inflammatory diseases. Nat. Rev. Immunol. 6, 205-217 (2006).

13. Tschernig, T. \& Pabst, R. Bronchus-associated lymphoid tissue (BALT) is not present in the normal adult lung but in different diseases. Pathobiology $68,1-8(2000)$

14. Pabst, R. \& Gehrke, I. Is the bronchus-associated lymphoid tissue (BALT) an integral structure of the lung in normal mammals, including humans? Am. J. Respir. Cell Mol. Biol. 3, 131-135 (1990).

15. Fagarasan, S., Muramatsu, M., Suzuki, K., Nagaoka, H., Hiai, H. \& Honjo, T. Critical roles of activation-induced cytidine deaminase in the homeostasis of gut flora. Science 298, 1424-1427 (2002).

16. Drayton, D.L., Liao, S., Mounzer, R.H. \& Ruddle, N.H. Lymphoid organ development: from ontogeny to neogenesis. Nat. Immunol. 7, 344-353 (2006).

17. Carragher, D.M., Rangel-Moreno, J. \& Randall, T.D. Ectopic lymphoid tissues and local immunity. Semin. Immunol. 20, 26-42 (2008).

18. Salomonsson, S., Larsson, P., Tengner, P., Mellquist, E., Hjelmstrom, P. \& Wahren-Herlenius, $M$. Expression of the $B$ cell-attracting chemokine CXCL13 in the target organ and autoantibody production in ectopic lymphoid tissue in the chronic inflammatory disease Sjogren's syndrome. Scand. J. Immunol. 55, 336-342 (2002).

19. Rangel-Moreno, J., Hartson, L., Navarro, C., Gaxiola, M., Selman, M. \& Randall, T.D. Inducible bronchus-associated lymphoid tissue (BBALT) in patients with pulmonary complications of rheumatoid arthritis. J. Clin. Invest. 116, 3183-3194 (2006).

20. Marchal-Somme, J. et al. Cutting edge: nonproliferating mature immune cells form a novel type of organized lymphoid structure in idiopathic pulmonary fibrosis. J. Immunol. 176, 5735-5739 (2006).

21. Gregorio, A. et al. Lymphoid neogenesis in juvenile idiopathic arthritis correlates with ANA positivity and plasma cells infiltration. Rheumatology (Oxford) 46, 308-313 (2007).

22. Weyand, C.M. \& Goronzy, J.J. Ectopic germinal center formation in rheumatoid synovitis. Ann. NY Acad. Sci. 987, 140-149 (2003).

23. Sato, A., Hayakawa, H., Uchiyama, H. \& Chida, K. Cellular distribution of bronchus-associated lymphoid tissue in rheumatoid arthritis. Am. J. Respir. Crit. Care Med. 154 (6 Pt 1), 1903-1907 (1996).

24. Elliot, J.G., Jensen, C.M., Mutavdzic, S., Lamb, J.P., Carroll, N.G. \& James, A.L. Aggregations of lymphoid cells in the airways of nonsmokers, smokers, and subjects with asthma. Am. J. Respir. Crit. Care Med. 169, 712-718 (2004).

25. Lee, J.J. et al. Interleukin-5 expression in the lung epithelium of transgenic mice leads to pulmonary changes pathognomonic of asthma. J. Exp. Med. 185, 2143-2156 (1997).

26. Richmond, I., Pritchard, G.E., Ashcroft, T., Avery, A., Corris, P.A. \& Walters, E.H. Bronchus associated lymphoid tissue (BALT) in human lung: its distribution in smokers and non-smokers. Thorax 48, 1130-1134 (1993).

27. van der Strate, B.W. et al. Cigarette smoke-induced emphysema: a role for the B cell? Am. J. Respir. Crit. Care Med. 173, 751-758 (2006).

28. Hogg, J.C. et al. The nature of small-airway obstruction in chronic obstructive pulmonary disease. N Engl. J. Med. 350, 2645-2653 (2004).

29. Moyron-Quiroz, J.E. et al. Role of inducible bronchus associated lymphoid tissue (BALT) in respiratory immunity. Nat. Med. 10, 927-934 (2004).

30. Halle, S. et al. Induced bronchus-associated lymphoid tissue serves as a general priming site for T cells and is maintained by dendritic cells. J. Exp. Med. 206, 2593-2601 (2009).

31. Luhrmann, A., Tschernig, T. \& Pabst, R. Stimulation of bronchusassociated lymphoid tissue in rats by repeated inhalation of aerosolized lipopeptide MALP-2. Pathobiology 70, 266-269 (2002).

32. Banfi, A. et al. Development of bronchus-associated lymphoid tissue hyperplasia following lipopolysaccharide-induced lung inflammation in rats. Exp. Lung Res. 35, 186-197 (2009).

33. Lorenz, R.G., Chaplin, D.D., McDonald, K.G., McDonough, J.S. \& Newberry, R.D. Isolated lymphoid follicle formation is inducible and dependent upon lymphotoxin-sufficient B lymphocytes, lymphotoxin beta receptor, and TNF receptor I function. J. Immunol. 170, 5475-5482 (2003).

34. Yoshida, H. et al. Expression of alpha(4)beta(7) integrin defines a distinct pathway of lymphoid progenitors committed to T cells, fetal intestinal 
lymphotoxin producer, NK, and dendritic cells. J. Immunol. 167, 2511-2521 (2001)

35. Tsuji, M. et al. Requirement for lymphoid tissue-inducer cells in isolated follicle formation and T cell-independent immunoglobulin A generation in the gut. Immunity 29, 261-271 (2008).

36. Yokota, Y. et al. Development of peripheral lymphoid organs and natural killer cells depends on the helix-loop-helix inhibitor Id2. Nature 397, 702-706 (1999).

37. Eberl, G. \& Littman, D.R. The role of the nuclear hormone receptor RORgammat in the development of lymph nodes and Peyer's patches. Immunol. Rev. 195, 81-90 (2003).

38. Fukuyama, S. et al. Initiation of NALT organogenesis is independent of the IL-7R, LT[beta]R, and NIK signaling pathways but requires the Id2 gene and CD3-CD4+CD45+ cells. Immunity 17, 31-40 (2002).

39. Marinkovic, T. et al. Interaction of mature $\mathrm{CD} 3+\mathrm{CD} 4+\mathrm{T}$ cells with dendritic cells triggers the development of tertiary lymphoid structures in the thyroid. J. Clin. Invest. 116, 2622-2632 (2006).

40. Mebius, R.E. Organogenesis of lymphoid tissues. Nat. Rev. Immunol. 3 , 292-303 (2003).

41. Ansel, K.M. et al. A chemokine-driven positive feedback loop organizes lymphoid follicles. Nature 406, 309-314 (2000).

42. Lee, M.K. IV et al. T-reg mediated suppression of the allograft response in the draining lymph node. Transplantation 81, 1063-1066 (2006).

43. Rangel-Moreno, J., Moyron-Quiroz, J.E., Hartson, L., Kusser, K. \& Randall, T.D. Pulmonary expression of CXC chemokine ligand 13, CC chemokine ligand 19, and CC chemokine ligand 21 is essential for local immunity to influenza. Proc. Natl. Acad. Sci. USA 104, 10577-10582 (2007).

44. Scheu, S., Alferink, J., Potzel, T., Barchet, W., Kalinke, U. \& Pfeffer, K. Targeted disruption of LIGHT causes defects in costimulatory $T$ cell activation and reveals cooperation with lymphotoxin beta in mesenteric lymph node genesis. J. Exp. Med. 195, 1613-1624 (2002).

45. Nishikawa, S., Honda, K., Vieira, P. \& Yoshida, H. Organogenesis of peripheral lymphoid organs. Immunol. Rev. 195, 72-80 (2003).

46. Drayton, D.L., Ying, X., Lee, J., Lesslauer, W. \& Ruddle, N.H. Ectopic LT alpha beta directs lymphoid organ neogenesis with concomitant expression of peripheral node addressin and a HEV-restricted sulfotransferase. J. Exp. Med. 197, 1153-1163 (2003).

47. Ngo, V.N. et al. Lymphotoxin alpha/beta and tumor necrosis factor are required for stromal cell expression of homing chemokines in B and T cell areas of the spleen. J. Exp. Med. 189, 403-412 (1999).

48. Ying, X., Chan, K., Shenoy, P., Hill, M. \& Ruddle, N.H. Lymphotoxin plays a crucial role in the development and function of nasal-associated lymphoid tissue through regulation of chemokines and peripheral node addressin. Am. J. Pathol. 166, 135-146 (2005).

49. Xu, B. et al. Lymphocyte homing to bronchus-associated lymphoid tissue (BALT) is mediated by L-selectin/PNAd, alpha4beta1 integrin/NCAM-1, and LFA-1 adhesion pathways. J. Exp. Med. 197, 1255-1267 (2003).

50. Kawamata, N. et al. Expression of endothelia and lymphocyte adhesion molecules in bronchus-associated lymphoid tissue (BALT) in adult human lung. Respir. Res. 10, 97 (2009).

51. Takemura, S. et al. Lymphoid neogenesis in rheumatoid synovitis. J. Immunol. 167, 1072-1080 (2001).

52. Manzo, A. et al. Systematic microanatomical analysis of CXCL13 and CCL21 in situ production and progressive lymphoid organization in rheumatoid synovitis. Eur. J. Immunol. 35, 1347-1359 (2005).

53. De Togni, P. et al. Abnormal development of peripheral lymphoid organs in mice deficient in lymphotoxin. Science 264, 703-707 (1994).

54. Banks, T.A. et al. Lymphotoxin-alpha-deficient mice. Effects on secondary lymphoid organ development and humoral immune responsiveness. J. Immunol. 155, 1685-1693 (1995).

55. Vuillemenot, B.R., Rodriguez, J.F. \& Hoyle, G.W. Lymphoid tissue and emphysema in the lungs of transgenic mice inducibly expressing tumor necrosis factor-\{alpha\}. Am. J. Respir. Cell Mol. Biol. 30, 438-448 (2004).

56. Goya, S. et al. Sustained interleukin- 6 signalling leads to the development of lymphoid organ-like structures in the lung. J. Pathol. 200, 82-87 (2003).

57. Legler, D.F., Loetscher, M., Roos, R.S., Clark-Lewis, I., Baggiolini, M. \& Moser, B. B cell-attracting chemokine 1, a human CXC chemokine expressed in lymphoid tissues, selectively attracts $B$ lymphocytes via BLR1/CXCR5. J. Exp. Med. 187, 655-660 (1998).

58. Luther, S.A., Lopez, T., Bai, W., Hanahan, D. \& Cyster, J.G. BLC expression in pancreatic islets causes $B$ cell recruitment and lymphotoxindependent lymphoid neogenesis. Immunity 12, 471-481 (2000).
59. Luther, S.A., Ansel, K.M. \& Cyster, J.G. Overlapping roles of CXCL13, interleukin 7 receptor alpha, and CCR7 ligands in lymph node development. J. Exp. Med. 197, 1191-1198 (2003).

60. Luther, S.A., Tang, H.L., Hyman, P.L., Farr, A.G. \& Cyster, J.G. Coexpression of the chemokines ELC and SLC by T zone stromal cells and deletion of the ELC gene in the plt/plt mouse. Proc. Natl Acad. Sci. USA 97, 12694-12699 (2000).

61. Marchal-Somme, J. et al. Dendritic cells accumulate in human fibrotic interstitial lung disease. Am. J. Respir. Crit. Care Med. 176, 1007-1014 (2007).

62. Fukuyama, S. et al. Cutting edge: uniqueness of lymphoid chemokine requirement for the initiation and maturation of nasopharynx-associated Iymphoid tissue organogenesis. J. Immunol. 177, 4276-4280 (2006).

63. Gribar, S.C., Anand, R.J., Sodhi, C.P. \& Hackam, D.J. The role of epithelial Toll-like receptor signaling in the pathogenesis of intestinal inflammation. J. Leukoc. Biol. 83, 493-498 (2008).

64. Sha, Q., Truong-Tran, A.Q., Plitt, J.R., Beck, L.A. \& Schleimer, R.P. Activation of airway epithelial cells by toll-like receptor agonists. Am. J. Respir. Cell Mol. Biol. 31, 358-364 (2004).

65. Emery, J.L. \& Dinsdale, F. The postnatal development of lymphoreticular aggregates and lymph nodes in infants' lungs. J. Clin. Pathol. 26, 539-545 (1973).

66. Tschernig, T., Kleemann, W.J. \& Pabst, R. Bronchus-associated lymphoid tissue (BALT) in the lungs of children who had died from sudden infant death syndrome and other causes. Thorax 50, 658-660 (1995).

67. Gould, S.J. \& Isaacson, P.G. Bronchus-associated lymphoid tissue (BALT) in human fetal and infant lung. J. Pathol. 169, 229-234 (1993).

68. Delventhal, S., Hensel, A., Petzoldt, K. \& Pabst, R. Effects of microbial stimulation on the number, size and activity of bronchus-associated lymphoid tissue (BALT) structures in the pig. Int. J. Exp. Pathol. 73, 351-357 (1992).

69. Meuwissen, H.J. \& Hussain, M. Bronchus-associated lymphoid tissue in human lung: correlation of hyperplasia with chronic pulmonary disease. Clin. Immunol. Immunopathol. 23, 548-561 (1982).

70. Cupedo, T., Jansen, W., Kraal, G. \& Mebius, R.E. Induction of secondary and tertiary lymphoid structures in the skin. Immunity 21, 655-667 (2004).

71. Phipps, S., Hansbro, N., Lam, C.E., Foo, S.Y., Matthaei, K.I. \& Foster, P.S Allergic sensitization is enhanced in early life through toll-like receptor 7 activation. Clin. Exp. Allergy 39, 1920-1928 (2009).

72. Luhrmann, A., Grote, K., Stephan, M., Tschernig, T. \& Pabst, R. Local pulmonary immune stimulation by the Toll-like receptor 2 and 6 ligand MALP-2 in rats is age dependent. Immunol. Lett. 108, 167-173 (2007).

73. Pabst, R., Durak, D., Roos, A., Luhrmann, A. \& Tschernig, T. TLR2/6 stimulation of the rat lung: effects on lymphocyte subsets, natural killer cells and dendritic cells in different parts of the air-conducting compartments and at different ages. Immunology 126, 132-139 (2009).

74. Moyron-Quiroz, J.E. et al. Persistence and responsiveness of immunologic memory in the absence of secondary lymphoid organs. Immunity 25, 643-654 (2006).

75. Steinman, R.M. Dendritic cells: understanding immunogenicity. Eur. J. Immunol. 37 (Suppl 1), S53-S60 (2007).

76. Beaty, S.R., Rose, C.E. Jr \& Sung, S.S. Diverse and potent chemokine production by lung CD11bhigh dendritic cells in homeostasis and in allergic lung inflammation. J. Immunol. 178, 1882-1895 (2007).

77. Van Pottelberge, G.R. et al. Plasmacytoid dendritic cells in pulmonary Iymphoid follicles of patients with COPD. Eur. Respir. J., published online 29 March 2010 (doi:10.1183/09031936.00140409).

78. Sakaguchi, S., Sakaguchi, N., Asano, M., Itoh, M. \& Toda, M. Immunologic self-tolerance maintained by activated T cells expressing IL-2 receptor alpha-chains (CD25). Breakdown of a single mechanism of self-tolerance causes various autoimmune diseases. J. Immunol. 155, 1151-1164 (1995).

79. Cederbom, L., Hall, H. \& Ivars, F. CD4+CD25+ regulatory T cells downregulate co-stimulatory molecules on antigen-presenting cells. Eur. J. Immunol. 30, 1538-1543 (2000).

80. Misra, N., Bayry, J., Lacroix-Desmazes, S., Kazatchkine, M.D. \& Kaveri, S.V. Cutting edge: human CD4+CD25+T cells restrain the maturation and antigen-presenting function of dendritic cells. J. Immunol. 172, 4676-4680 (2004).

81. Serra, P. et al. CD40 ligation releases immature dendritic cells from the control of regulatory CD4+CD25+ T cells. Immunity 19, 877-889 (2003).

82. Thornton, A.M. \& Shevach, E.M. CD4+CD25+ immunoregulatory T cells suppress polyclonal $\mathrm{T}$ cell activation in vitro by inhibiting interleukin 2 production. J. Exp. Med. 188, 287-296 (1998). 


\section{REVIEW}

83. Nakamura, K., Kitani, A. \& Strober, W. Cell contact-dependent immunosuppression by CD4(+)CD25(+) regulatory T cells is mediated by cell surface-bound transforming growth factor beta. J. Exp. Med. 194, 629-644 (2001).

84. Ueha, S. et al. CCR7 mediates the migration of Foxp3+ regulatory T cells to the paracortical areas of peripheral lymph nodes through high endothelial venules. J. Leukoc. Biol. 82, 1230-1238 (2007).

85. Tirouvanziam, R. et al. Ex vivo development of functional human lymph node and bronchus-associated lymphoid tissue. Blood 99, 2483-2489 (2002).

86. Heier, I. et al. Bronchial response pattern of antigen presenting cells and regulatory $T$ cells in children less than 2 years of age. Thorax $63,703-709$ (2008).

87. Tsuji, M. et al. Preferential generation of follicular B helper T cells from Foxp3+ T cells in gut Peyer's patches. Science 323, 1488-1492 (2009).

88. McDermott, M.R. \& Bienenstock, J. Evidence for a common mucosal immunologic system. I. Migration of B immunoblasts into intestinal, respiratory, and genital tissues. J. Immunol. 122, 1892-1898 (1979).

89. Rudzik, R., Clancy, R.L., Perey, D.Y., Day, R.P. \& Bienenstock, J. Repopulation with IgA-containing cells of bronchial and intestinal lamina propria after transfer of homologous Peyer's patch and bronchial lymphocytes. J. Immunol. 114, 1599-1604 (1975).

90. Kocks, J.R. et al. Chemokine receptor CCR7 contributes to a rapid and efficient clearance of lytic murine gamma-herpes virus 68 from the lung, whereas bronchus-associated lymphoid tissue harbors virus during latency. J. Immunol. 182, 6861-6869 (2009).

91. Wiley, J.A. et al. Inducible bronchus-associated lymphoid tissue elicited by a protein cage nanoparticle enhances protection in mice against diverse respiratory viruses. PLoS One 4, e7142 (2009).

92. Norton, E.B., Clements, J.D., Voss, T.G. \& Cardenas-Freytag, L. Prophylactic administration of bacterially derived immunomodulators improves the outcome of influenza virus infection in a murine model. J. Virol. 84, 2983-2995 (2010). 